\title{
The noncommutative Poisson bracket and the deformation of the family algebras
}

\author{
Zhaoting Wei \\ Department of Mathematics, Indiana University, \\ 831 E 3rd Street, Bloomington, IN 47405, USA ${ }^{*}$ \\ (Dated: submitted on April 4, 2015, revised on June 7, 2018)
}

The family algebras are introduced by A.A. Kirillov in 2000. In this paper we study the noncommutative Poisson bracket $P$ on the classical family algebra $\mathcal{C}_{\tau}(\mathfrak{g})$. We show that $P$ controls the first-order 1-parameter formal deformation from $\mathcal{C}_{\tau}(\mathfrak{g})$ to $\mathcal{Q}_{\tau}(\mathfrak{g})$ where the later is the quantum family algebra. Moreover we will prove that the noncommutative Poisson bracket is in fact a Hochschild 2-coboundary and therefore the deformation is infinitesimally trivial. In the last part of this paper we discuss the relation between Mackey's analogue and the quantization problem of the family algebras.

\section{INTRODUCTION}

The classical and quantum family algebras are introduced by A. A. Kirillov in the year 2000 in [14] and [15] as a new tool to study representation theory of Lie algebras. They have some interesting properties and various applications in Lie theory, representation theory and mathematical physics.

Let us briefly review the definitions of them. Let $\mathfrak{g}$ be a finite dimensional complex Lie algebra, $S(\mathfrak{g})$ and $U(\mathfrak{g})$ be the symmetric algebra and the universal enveloping algebra of $\mathfrak{g}$, respectively. Let $G$ be a connected and simply connected Lie group with $\operatorname{Lie}(G)=\mathfrak{g} . G$ has adjoint actions Ad on $S(\mathfrak{g})$ and $U(\mathfrak{g})$.

On the other hand, let $V_{\tau}$ be a finite dimensional complex representation of $\mathfrak{g}$. Then $\tau$ gives rise to a representation of $G$. Hence $G$ has a natural action on $\operatorname{End}_{\mathbb{C}} V_{\tau}$ :

$$
\forall A \in \operatorname{End}_{\mathbb{C}} V_{\tau}, g \in G, g \cdot A:=\tau(g) A \tau(g)^{-1}
$$

and therefore $G$ has natural diagonal actions on $\operatorname{End}_{\mathbb{C}} V_{\tau} \otimes_{\mathbb{C}} S(\mathfrak{g})$ and $\operatorname{End}_{\mathbb{C}} V_{\tau} \otimes_{\mathbb{C}} U(\mathfrak{g})$.

Now we come to the definition of the family algebras, see [14] and [15]:

Definition 1.1 (The family algebras). The classical family algebra is defined to be:

$$
\mathcal{C}_{\tau}(\mathfrak{g}):=\left(\operatorname{End}_{\mathbb{C}} V_{\tau} \otimes_{\mathbb{C}} S(\mathfrak{g})\right)^{G} .
$$

The quantum family algebra is defined to be:

$$
\mathcal{Q}_{\tau}(\mathfrak{g}):=\left(\operatorname{End}_{\mathbb{C}} V_{\tau} \otimes_{\mathbb{C}} U(\mathfrak{g})\right)^{G} .
$$

Remark 1.1. Actually the classical family algebra is a generalization of the invariant polynomial algebra $I(\mathfrak{g})$ and the quantum family algebra is a generalization of the center of the universal enveloping algebra $Z(\mathfrak{g})$, see Example 2.1 below.

Kirillov proves that $\mathcal{C}_{\tau}(\mathfrak{g})$ and $\mathcal{Q}_{\tau}(\mathfrak{g})$ are indeed algebras, that is, they are closed under multiplications. A.A. Kirillov [14], [15], A. Joseph [13], N. Rozhkovskaya [21] and M. Tai [22] have found various relations between family algebras and the representations of $\mathfrak{g}$. For other applications, N. Higson relates family algebras with the admissible representations of complex semisimple Lie groups in [12]

Remark 1.2. The quantum family algebra is called the relative Yangian in [13].

In this paper, we study the family algebras from another viewpoint. It is well-known that we have a Poisson bracket on $S(\mathfrak{g})$ (see [16]): Let $X_{i}$ be a basis of $\mathfrak{g}$ and $c_{i j}^{k}$ be the structure constant with respect to the basis $X_{i}$, then for any $a, b \in S(\mathfrak{g})$, the Poisson bracket is defined to be

$$
\{a, b\}:=c_{i j}^{k} X_{k} \cdot \partial^{i} a \cdot \partial^{j} b
$$

where $\partial^{i}$ denotes the partial derivative with respect to $X_{i}$.

Now we can define the noncommutative Poisson bracket on the classical family algebra:

Definition 1.2 (The noncommutative Poisson bracket on $\mathcal{C}_{\tau}(\mathfrak{g})$ ). Let $\mathcal{A}, \mathcal{B} \in \mathcal{C}_{\tau}(\mathfrak{g}), \mathcal{A}=A_{i} \otimes a^{i}, \mathcal{B}=B_{j} \otimes b^{j}$. We define the noncommutative Poisson bracket $P$ as follows:

$$
\{\mathcal{A}, \mathcal{B}\}:=A_{i} B_{j} \otimes\left\{a^{i}, b^{j}\right\} .
$$


In this paper we will study the properties of the noncommutative Poisson bracket (for short, Poisson bracket) on $\mathcal{C}_{\tau}(\mathfrak{g})$. The following are two important results we get:

- The Poisson bracket on $\mathcal{C}_{\tau}(\mathfrak{g})$ characterize the first-order 1-parameter formal deformations from $\mathcal{C}_{\tau}(\mathfrak{g})$ to $\mathcal{Q}_{\tau}(\mathfrak{g})$, just as the Poisson bracket on $S(\mathfrak{g})$ characterize the first-order 1-parameter formal deformations from $S(\mathfrak{g})$ to $U(\mathfrak{g})$, see Proposition 4.6

- In the Hochschild cochain complex of $\mathcal{C}_{\tau}(\mathfrak{g})$, the Poisson bracket is a 2-coboundary. In fact we can explicitly find a Hochschild 1-cochain $\nabla$ which maps to the Poisson bracket under the Hochschild differential, see Theorem 5.4

It is expected that this result has applications in representation theory as well as mathematical physics. For example, it may help us find a quantization map $\mathcal{C}_{\tau}(\mathfrak{g}) \rightarrow \mathcal{Q}_{\tau}(\mathfrak{g})$, as proposed by Higson in [12]. See SectionVI for details.

This paper is organized as follows: In Section [I] we review the family algebras, in Section $\llbracket$ we study the first properties of the noncommutative Poisson bracket $P$ on the classical family algebra. In Section [V] we give the relation between $P$ and the 1-parameter formal deformation from $\mathcal{C}_{\tau}(\mathfrak{g})$ to $\mathcal{Q}_{\tau}(\mathfrak{g})$. In Section $\square$ we prove that the noncommutative Poisson bracket $P$ is a Hochschild 2-coboundary and therefore the deformation is infinitesimally trivial. In Section VI we talk about the quantization problem of the family algebras. In the three appendices we summarize the results on Hochschild cohomology, Gerstenhaber bracket and their relation to the deformation theory.

Remark 1.3. Although Kirillov and Higson in [14], [15] and [12] require the Lie algebra $\mathfrak{g}$ to be semisimple and the representation $\tau$ to be irreducible, in this paper we do not need this restriction, except for Section VI]

\section{Conventions}

Let $\mathfrak{g}$ be a finite dimensional complex Lie algebra. We use $\left\{X_{i}\right\}$ for a basis for $\mathfrak{g}$ and $c_{i j}^{k}$ the structure constant of $\mathfrak{g}$ with respect to $\left\{X_{i}\right\}$.

We use the letters in lower case $a, b$ or $a_{i}, b_{j}$ etc. for the elements in $S(\mathfrak{g})$. The symbol $\partial^{k}$ denotes the partial derivative of elements in $S(\mathfrak{g})$ with respect to $X_{k}$.

Let $V_{\tau}$ be a finite representation of $\mathfrak{g}$, we use the capital letter $A, B$ or $A_{i}, B_{j}$ etc. to represent the elements in $\operatorname{End}\left(V_{\tau}\right)$.

The calligraphic letters $\mathcal{A}, \mathcal{B}$ etc. stand for elements in the family algebras $\mathcal{C}_{\tau}(\mathfrak{g})$ and $\mathcal{Q}_{\tau}(\mathfrak{g})$.

In this paper we frequently use the Einstein summation convention: $A_{i} \otimes a^{i}$ means taking sum with respect to $i$

\section{A BRIEF INTRODUCTION TO THE FAMILY ALGEBRAS}

We give a quick review of family algebras in this section. Most of the materials in this section can be found in [14] and [15].

First of all, we use the following notation-definition

\section{Definition 2.3.}

$$
\begin{aligned}
& \widetilde{\mathcal{C}_{\tau}}(\mathfrak{g}):=\operatorname{End}_{\mathbb{C}} V_{\tau} \otimes_{\mathbb{C}} S(\mathfrak{g}), \\
& \widetilde{\mathcal{Q}_{\tau}}(\mathfrak{g}):=\operatorname{End}_{\mathbb{C}} V_{\tau} \otimes_{\mathbb{C}} U(\mathfrak{g}) .
\end{aligned}
$$

$\widetilde{\mathcal{C}_{\tau}}(\mathfrak{g})$ and $\widetilde{\mathcal{Q}_{\tau}}(\mathfrak{g})$ consist of matrices with entries in $S(\mathfrak{g})$ and $U(\mathfrak{g})$, respectively. Therefore they are algebras in a natural way: for any $A_{i} \otimes a^{i}, B_{j} \otimes b^{j} \in \widetilde{\mathcal{C}_{\tau}}(\mathfrak{g})\left(\right.$ or $\left.\widetilde{\mathcal{Q}_{\tau}}(\mathfrak{g})\right)$, their product is given by the following formula:

$$
\left(A_{i} \otimes a^{i}\right) \cdot\left(B_{j} \otimes b^{j}\right):=A_{i} B_{j} \otimes a^{i} b^{j}
$$

Caution 2.1. $\widetilde{\mathcal{C}_{\tau}}(\mathfrak{g})$ and $\widetilde{\mathcal{Q}_{\tau}}(\mathfrak{g})$ are non-commutative in general.

The following simple result will be frequently used: 
Lemma 2.1. In both $\widetilde{\mathcal{C}_{\tau}}(\mathfrak{g})$ and $\widetilde{\mathcal{Q}_{\tau}}(\mathfrak{g})$, the matrix component and the $S(\mathfrak{g})$ component always commute. In more detail, for any $A_{i} \otimes a^{i}, B_{j} \otimes b^{j} \in \widetilde{\mathcal{C}_{\tau}}(\mathfrak{g})\left(\right.$ or $\left.\widetilde{\mathcal{Q}_{\tau}}(\mathfrak{g})\right)$, we have

$$
\begin{aligned}
\left(A_{i} \otimes a^{i}\right) \cdot\left(B_{j} \otimes b^{j}\right) & =A_{i} B_{j} \otimes a^{i} b^{j} \\
& =\left(I d \otimes a^{i}\right) \cdot\left(A_{i} B_{j} \otimes b^{j}\right) \\
& =\left(A_{i} B_{j} \otimes a^{i}\right) \cdot\left(I d \otimes b^{j}\right) .
\end{aligned}
$$

Proof. It is obvious.

By Definition 1.1 we know

$$
\left.\mathcal{C}_{\tau}(\mathfrak{g})=\widetilde{\mathcal{C}_{\tau}}(\mathfrak{g})^{G} \text { and } \mathcal{Q}_{\tau}(\mathfrak{g}):=\widetilde{\mathcal{Q}_{\tau}}(\mathfrak{g})\right)^{G}
$$

Now we show that the Lie group action can be reduced to the Lie algebra action.

Proposition 2.2 (The infinitesimal test for classical family algebra, [14] Section 1). Let $A_{i} \otimes a^{i} \in \widetilde{\mathcal{C}_{\tau}}(\mathfrak{g})$, then $A_{i} \otimes a^{i} \in \mathcal{C}_{\tau}(\mathfrak{g})$ if and only if

$$
\forall X \in \mathfrak{g},\left[\tau(X), A_{i}\right] \otimes a^{i}+A_{i} \otimes\left\{X, a^{i}\right\}=0 .
$$

In other words,

$$
\forall X \in \mathfrak{g},\left[\tau(X), A_{i}\right] \otimes a^{i}=A_{i} \otimes\left\{a^{i}, X\right\}
$$

Proof. By definition 1.1 we know that $A_{i} \otimes a^{i} \in \mathcal{C}_{\tau}(\mathfrak{g})$ if and only if:

$$
\tau(g) \circ A_{i} \circ \tau(g)^{-1} \otimes(\operatorname{ad} g) a^{i}=A_{i} \otimes a^{i} .
$$

It is well-known that the adjoint action of $\mathfrak{g}$ on $S(\mathfrak{g})$ is exactly the Poisson bracket. As a result, Equation (7) and $(8)$ are infinitesimal versions of the above equation. Since $G$ is connected and simply connected, they are equivalent to the invariance under the action of $G$.

Similarly we have

Proposition 2.3 (The infinitesimal test for quantum family algebra, [14] Section 1). Let $A_{i} \otimes a^{i} \in \widetilde{\mathcal{Q}_{\tau}}(\mathfrak{g})$, then $A_{i} \otimes a^{i} \in \mathcal{Q}_{\tau}(\mathfrak{g})$ if and only if

$$
\forall X \in \mathfrak{g},\left[\tau(X), A_{i}\right] \otimes a^{i}+A_{i} \otimes\left[X, a^{i}\right]=0 .
$$

In other words,

$$
\forall X \in \mathfrak{g},\left[\tau(X), A_{i}\right] \otimes a^{i}=A_{i} \otimes\left[a^{i}, X\right]
$$

Proof. Similar to the proof of Proposition 2.3.

Then we can prove the following result:

Corollary 2.4 (see also [14] and [15]). $\mathcal{C}_{\tau}(\mathfrak{g})$ and $\mathcal{Q}_{\tau}(\mathfrak{g})$ are subalgebras of $\widetilde{\mathcal{C}_{\tau}}(\mathfrak{g})$ and $\left.\widetilde{\mathcal{Q}_{\tau}}(\mathfrak{g})\right)$ respectively.

Proof. Let $A_{i} \otimes a^{i}$ and $B_{j} \otimes b^{j}$ be two elements in $\mathcal{C}_{\tau}(\mathfrak{g})$. Their product

$$
\left(A_{i} \otimes a^{i}\right) \cdot\left(B_{j} \otimes b^{j}\right)=A_{i} B_{j} \otimes a^{i} b^{j} .
$$

Now $\forall X \in \mathfrak{g}$,

$$
\begin{aligned}
{\left[\tau(X), A_{i} B_{j}\right] \otimes a^{i} b^{j} } & =\left[\tau(X), A_{i}\right] B_{j} \otimes a^{i} b^{j}+A_{i}\left[\tau(X), B_{j}\right] \otimes a^{i} b^{j} \\
& =\left(\left[\tau(X), A_{i}\right] \otimes a^{i}\right) \cdot\left(B_{j} \otimes b^{j}\right)+\left(A_{i} \otimes a^{i}\right) \cdot\left(\left[\tau(X), B_{j}\right] \otimes b^{j}\right) .
\end{aligned}
$$

The second equality is because of Lemma2.1 the matrix component always commutes with the $S(\mathfrak{g})$ component. Now by Proposition 2.2, we know

$$
\begin{aligned}
\text { the above formula } & =\left(A_{i} \otimes\left\{a^{i}, X\right\}\right) \cdot\left(B_{j} \otimes b^{j}\right)+\left(A_{i} \otimes a^{i}\right) \cdot B_{j} \otimes\left\{b^{j}, X\right\} \\
& =A_{i} B_{j} \otimes\left\{a^{i}, X\right\} b^{j}+A_{i} B_{j} \otimes a^{i}\left\{b^{j}, X\right\} \text { (Proposition2.2) } \\
& =A_{i} B_{j} \otimes\left\{a^{i} b^{j}, X\right\} .
\end{aligned}
$$


Hence we get

$$
A_{i} B_{j} \otimes a^{i} b^{j} \in \mathcal{C}_{\tau}(\mathfrak{g}) .
$$

In the same way we can show that if $A_{i} \otimes a^{i}$ and $B_{j} \otimes b^{j}$ are in $\mathcal{Q}_{\tau}(\mathfrak{g})$, then

$$
A_{i} B_{j} \otimes a^{i} b^{j} \in \mathcal{Q}_{\tau}(\mathfrak{g}) .
$$

It is not difficult to see that the family algebras contains non-zero elements. In fact, let $I(\mathfrak{g})=S(\mathfrak{g})^{\mathfrak{g}}$ be the invariant subalgebra of $S(\mathfrak{g})$ and $Z(\mathfrak{g})$ be the center of $U(\mathfrak{g})$. We have

Proposition 2.5 ([15]). I $(\mathfrak{g})$ embeds into $\mathcal{C}_{\tau}(\mathfrak{g})$ as scalar matrices

$$
\begin{aligned}
I(\mathfrak{g}) & \hookrightarrow \mathcal{C}_{\tau}(\mathfrak{g}) \\
a & \mapsto I d \otimes a .
\end{aligned}
$$

Similarly $Z(\mathfrak{g})$ embeds into $\mathcal{Q}_{\tau}(\mathfrak{g})$ as scalar matrices too.

Proof. It is obvious that $I(\mathfrak{g})$ embeds into $\widetilde{\mathcal{C}_{\tau}}(\mathfrak{g})$ as scalar matrices. Now by Proposition 2.2, it is easy to see that the image is contained in $\mathcal{C}_{\tau}(\mathfrak{g})$.

The proof for $Z(\mathfrak{g})$ and $\mathcal{Q}_{\tau}(\mathfrak{g})$ is the same.

Example 2.1. For any $\mathfrak{g}$, when the representation $\tau$ is the trivial representation, we see that $I(\mathfrak{g})=\mathcal{C}_{\tau}(\mathfrak{g})$ and $Z(\mathfrak{g})=\mathcal{Q}_{\tau}(\mathfrak{g})$.

Example 2.2. For $\mathfrak{g}=\operatorname{sl}(2, \mathbb{C})$ and $\{e, f, h\}$ be the standard basis of $\operatorname{sl}(2, \mathbb{C})$ which satisfies the commutation relation

$$
[e, f]=h,[h, e]=2 e,[h, f]=-2 f .
$$

Let $\tau$ be the 2-dimensional standard representation, we can find an element $M \in \mathcal{C}_{\tau}(\mathfrak{g})$ which is not in $I(\mathfrak{g})$. In fact

$$
M=\left(\begin{array}{cc}
\frac{h}{2} & f \\
e & -\frac{h}{2}
\end{array}\right)
$$

We can also find an element in $\mathcal{Q}_{\tau}(\mathfrak{g})$ with the same expression of $M$, see [14] and [15].

Remark 2.4. When $\tau$ is nontrivial irreducible and $\mathfrak{g}$ is semisimple, $I(\mathfrak{g})$ is not equal to $\mathcal{C}_{\tau}(\mathfrak{g})$ and $Z(\mathfrak{g})$ is not equal to $\mathcal{Q}_{\tau}(\mathfrak{g})$ either, see Corollary 5.3 below or [14].

\section{THE NONCOMMUTATIVE POISSON BRACKET ON $\widetilde{\mathcal{C}_{\tau}}(\mathfrak{g})$}

The noncommutative Poisson bracket on $\mathcal{C}_{\tau}(\mathfrak{g})$ in Definition 1.2 can be automatically extended to $\widetilde{\mathcal{C}_{\tau}}(\mathfrak{g})$ :

Definition 3.4. Let $\mathcal{A}, \mathcal{B} \in \widetilde{\mathcal{C}_{\tau}}(\mathfrak{g}), \mathcal{A}=A_{i} \otimes a^{i}, \mathcal{B}=B_{j} \otimes b^{j}$. We define the noncommutative Poisson bracket as follows:

$$
\{\mathcal{A}, \mathcal{B}\}:=A_{i} B_{j} \otimes\left\{a^{i}, b^{j}\right\}
$$

We will also denote the noncommutative Poisson bracket by $P$.

Remark 3.5. P. Xu gives a similar construction in [24] Example 2.2.

Caution 3.2. The Poisson bracket on $\widetilde{\mathcal{C}_{\tau}}(\mathfrak{g})$ is not anti-symmetric and does not satisfies the Leibniz rule and the Jacobi identity.

Nevertheless, J. Block and E. Getzler in 1992 give a definition of Poisson brackets on noncommutative algebras in [2] and we can prove that our noncommutative Poisson bracket $P$ satisfies the requirement of Poisson bracket in that sense:

Definition 3.5 ([2] Definition 1.1). A Poisson bracket on a (possibly noncommutative) algebra $A$ is a Hochschild 2-cocycle $P \in Z^{2}(A, A)$ such that $P \circ P \in C^{3}(A, A)$ is a 3-coboundary. In other words

$$
P \circ P \in B^{3}(A, A) \subset Z^{3}(A, A) \subset C^{3}(A, A) .
$$


For Hochschild cohomology see Appendix $\AA$ and for the definition of $P \circ P$ see Proposition B.1.

Remark 3.6. In Definition 3.5, we may take the condition $P \in Z^{2}(A, A)$ as a noncommutative Leibniz rule and $P \circ P \in B^{3}(A, A)$ as a noncommutative Jacobi identity. They together implies that $P$ can be lift to an associative product on $A$ up to order 3 , see Corollary C.2.

For our algebra $\widetilde{\mathcal{C}_{\tau}}(\mathfrak{g})$ and the Poisson bracket $P$ in Definition 3.4, first we can prove that $P$ is a 2-cocycle. We have the following proposition:

Proposition 3.1. For any $\mathcal{A}, \mathcal{B}, \mathcal{C} \in \widetilde{\mathcal{C}_{\tau}}(\mathfrak{g})$, we have

$$
\mathcal{A}\{\mathcal{B}, \mathcal{C}\}-\{\mathcal{A B}, \mathcal{C}\}+\{\mathcal{A}, \mathcal{B C}\}-\{\mathcal{A}, \mathcal{B}\} \mathcal{C}=0 .
$$

In other words, we have $d_{H} P=0$ where $d_{H}$ is the Hochschild differential operator. Therefore

$$
P \in Z^{2}\left(\widetilde{\mathcal{C}_{\tau}}(\mathfrak{g}), \widetilde{\mathcal{C}_{\tau}}(\mathfrak{g})\right) .
$$

Proof. Let $\mathcal{A}=A_{i} \otimes a^{i}, \mathcal{B}=B_{j} \otimes b^{j}$ and $\mathcal{C}=C_{k} \otimes c^{k}$. Recall that we have Lemma2.1, the matrix component and the $S(\mathfrak{g})$ component always commute. Then by the definition of the Poisson bracket we get

$$
\begin{aligned}
& \mathcal{A}\{\mathcal{B}, \mathcal{C}\}-\{\mathcal{A B}, \mathcal{C}\}+\{\mathcal{A}, \mathcal{B C}\}-\{\mathcal{A}, \mathcal{B}\} \mathcal{C} \\
= & A_{i} B_{j} C_{k} \otimes\left(a_{i}\left\{b_{j}, c_{k}\right\}-\left\{a_{i} b_{j}, c_{k}\right\}+\left\{a_{i}, b_{j} c_{k}\right\}-\left\{a_{i}, b_{j}\right\} c_{k}\right) .
\end{aligned}
$$

By the Leibniz rule of the (ordinary) Poisson bracket on $S(\mathfrak{g})$ we know that

$$
a_{i}\left\{b_{j}, c_{k}\right\}-\left\{a_{i} b_{j}, c_{k}\right\}+\left\{a_{i}, b_{j} c_{k}\right\}-\left\{a_{i}, b_{j}\right\} c_{k}=0 .
$$

Proposition 3.2. $P \circ P$ is a 3-coboundary. In other words, $P \circ P \in B^{3}\left(\widetilde{\mathcal{C}_{\tau}}(\mathfrak{g}), \widetilde{\mathcal{C}_{\tau}}(\mathfrak{g})\right)$.

Proof. In fact, we can define a 2-cochain $\Phi \in C^{2}\left(\widetilde{\mathcal{C}_{\tau}}(\mathfrak{g}), \widetilde{\mathcal{C}_{\tau}}(\mathfrak{g})\right)$ as follows: Let $\mathcal{A}=A \otimes a$ and $\mathcal{B}=B \otimes b$ (to simplify the notation we omit the super and sub-indices)

$$
\begin{aligned}
\Phi(\mathcal{A}, \mathcal{B}): & =A B \otimes \frac{1}{2} c_{i j}^{s} c_{k l}^{t} X_{s} \cdot X_{t} \cdot \partial^{i} \partial^{k} a \cdot \partial^{j} \partial^{l} b \\
& +A B \otimes \frac{1}{3} c_{k s}^{t} c_{j i}^{s} X_{t}\left(\partial^{k} \partial^{j} a \cdot \partial^{i} b+\partial^{i} a \cdot \partial^{k} \partial^{j} b\right) .
\end{aligned}
$$

Then we have

$$
P \circ P+\mathrm{d}_{\mathrm{H}} \Phi=0
$$

For any $\mathcal{A}, \mathcal{B}, \mathcal{C} \in \widetilde{\mathcal{C}_{\tau}}(\mathfrak{g})$, by Definition

$$
P \circ P(\mathcal{A}, \mathcal{B}, \mathcal{C})=\{\mathcal{A},\{\mathcal{B}, \mathcal{C}\}\}-\{\{\mathcal{A}, \mathcal{B}\}, \mathcal{C}\} .
$$

Let $\mathcal{A}=A \otimes a, \mathcal{B}=B \otimes b$ and $\mathcal{C}=C \otimes c$, then

$$
P \circ P(\mathcal{A}, \mathcal{B}, \mathcal{C})=A B C \otimes(\{a,\{b, c\}\}-\{\{a, b\}, c\}) .
$$

Now the problem reduces to $S(\mathfrak{g})$. We have the following lemma:

Lemma 3.3. We can define a 2 -cochain $\phi \in C^{2}(S(\mathfrak{g}), S(\mathfrak{g}))$ as follows: for any $a, b \in S(\mathfrak{g})$

$$
\phi(a, b):=\frac{1}{2} c_{i j}^{s} c_{k l}^{t} X_{s} \cdot X_{t} \cdot \partial^{i} \partial^{k} a \cdot \partial^{j} \partial^{l} b+\frac{1}{3} c_{k s}^{t} c_{j i}^{s} X_{t} \cdot\left(\partial^{k} \partial^{j} a \cdot \partial^{i} b+\partial^{i} a \cdot \partial^{k} \partial^{j} b\right) .
$$

Then for any $a, b, c \in S(\mathfrak{g})$ we have

$$
\{a,\{b, c\}\}-\{\{a, b\}, c\}+\left(d_{H} \phi\right)(a, b, c)=0 .
$$

Proof of Lemma 3.3 We can check it by hand using Jacobi identity. Another approach involves the star-porduct on $S(\mathfrak{g})$ and the general result of deformation theory and we defer it to Proposition 4.2 .

Now we have Lemma3.3. By abusing the notations we have $\Phi=\operatorname{Id} \otimes \phi$, then we immediately get $P \circ P+\mathrm{d}_{\mathrm{H}} \Phi=$ 0 . This finishes the proof of Proposition 3.2 
By Proposition 3.1 and 3.2 we know that the noncommutative Poisson bracket in Definition 3.4 is indeed a Poisson bracket in the sense of Definition 3.5 .

Before we move on, we need to prove that the Poisson bracket indeed maps $\mathcal{C}_{\tau}(\mathfrak{g}) \otimes \mathcal{C}_{\tau}(\mathfrak{g})$ to $\mathcal{C}_{\tau}(\mathfrak{g})$. That is the following proposition:

Proposition 3.4. For any $\mathcal{A}, \mathcal{B} \in \mathcal{C}_{\tau}(\mathfrak{g})$, we have that $\{\mathcal{A}, \mathcal{B}\}$ is still in $\mathcal{C}_{\tau}(\mathfrak{g})$. In other words, the noncommutative Poisson bracket in Definition 1.2 is well-defined.

Proof. We can proof this proposition by computation using Proposition 2.2 and the definition of the noncommutative Poisson bracket $P$. In Section $\nabla$ we will give another proof using a different construction of $P$. See Corollary 5.7

\section{THE NONCOMMUTATIVE POISSON BRACKET AND THE 1-PARAMETER FORMAL DEFORMATION OF $\widetilde{\mathcal{C}_{\tau}}(\mathfrak{g})$}

In this section we will show that the Poisson bracket plays an essential role in the 1-parameter formal deformation of $\widetilde{\mathcal{C}_{\tau}}(\mathfrak{g})$.

\section{A. A quick review of the 1-parameter formal deformation from $S(\mathfrak{g})$ to $U(\mathfrak{g})$ and the Poisson bracket}

Before studying the deformation of $\widetilde{\mathcal{C}_{\tau}}(\mathfrak{g})$, let us first review the corresponding theory of $S(\mathfrak{g})$ and $U(\mathfrak{g})$. It is well-known that

$$
S(\mathfrak{g})=\mathrm{T}(\mathfrak{g}) /(X \otimes Y-Y \otimes X)
$$

and

$$
U(\mathfrak{g})=\mathrm{T}(\mathfrak{g}) /(X \otimes Y-Y \otimes X-[X, Y])
$$

where $T(\mathfrak{g})$ is the tensor algebra of $\mathfrak{g}$.

Moreover, we consider the algebra

$$
U_{t}(\mathfrak{g})=\mathrm{T}(\mathfrak{g}) /(X \otimes Y-Y \otimes X-t[X, Y]) .
$$

For $t \neq 0$ all the algebras $U_{t}(\mathfrak{g})$ are isomorphic to $U(\mathfrak{g})$, and when $t=0, U_{0}(\mathfrak{g})$ is isomorphic to $S(\mathfrak{g}) . t$ is called the deformation parameter.

Remark 4.7. For more details on the 1-parameter formal deformation of an associative algebra see Appendix C

We have the Poincaré-Birkhoff-Witt map ( $\left.\mathrm{I}_{\mathrm{PBW}}\right)$ from $S(\mathfrak{g})$ to $U_{t}(\mathfrak{g})$ given by:

$$
\begin{aligned}
\mathrm{I}_{\mathrm{PBW}}: S(\mathfrak{g}) & \longrightarrow U_{t}(\mathfrak{g}) \\
X_{1} X_{2} \ldots X_{k} & \longmapsto \sum_{\sigma \in S_{k}} \frac{1}{k !} X_{\sigma(1)} X_{\sigma(2)} \ldots X_{\sigma(k)} .
\end{aligned}
$$

The Poincaré-Birkhoff-Witt theorem (see [17]) tells us that the above map I vector spaces.

Remark 4.8. The map $\mathrm{I}_{\mathrm{PBW}}$ is not an algebraic isomorphism unless $\mathfrak{g}$ is an abelian Lie algebra or $t=0$.

Therefore we have the following definition

Definition 4.6. The map I $\mathrm{IBB}_{\mathrm{W}}$ pulls back the multiplication of $U_{t}(\mathfrak{g})$ to $S(\mathfrak{g})$ and we call it the star-product on $S(\mathfrak{g})$, denoted by $*_{t}$. For any $a, b \in S(\mathfrak{g})$

$$
a *_{t} b:=\mathrm{I}_{\mathrm{PBW}}^{-1}\left(\mathrm{I}_{\mathrm{PBW}}(a) \cdot \mathrm{I}_{\mathrm{PBW}}(b)\right) .
$$

In particular we denote $*_{1}$ simply as $*$. When $t=0$ the star-product reduces to the original production on $S(\mathfrak{g})$. Obviously $*_{t}$ satisfies the associativity law because the multiplication on $U_{t}(\mathfrak{g})$ is associative.

Now by definition, the map $\mathrm{I}_{\mathrm{PB}}$ gives an algebraic isomorphism

$$
\mathrm{I}_{\mathrm{PBW}}:\left(S(\mathfrak{g}), *_{t}\right) \stackrel{\sim}{\longrightarrow}\left(U_{t}(\mathfrak{g}), \cdot\right) .
$$

Therefore we can identify $U_{t}(\mathfrak{g})$ with $\left(S(\mathfrak{g}), *_{t}\right)$, especially we can identify $U(\mathfrak{g})$ with $(S(\mathfrak{g}), *)$. 
Remark 4.9. Our star-product $*_{t}$ is not exactly the same as the sart-product constructed by Kontsevich in [18] Section 8. Nevertheless, they give isomorphic algebra structures on $S(\mathfrak{g})$.

The star-product $*_{t}$ depends on the deformation parameter $t$. In fact we can write the first few terms of $*_{t}$.

Proposition 4.1 ([11] Section 3). We can write $*_{t}$ as

$$
a *_{t} b=a b+\frac{t}{2}\{a, b\}+O\left(t^{2}\right) .
$$

Proof. This result is well-known and can be found in, say, [11] Section 3.

In other words, the Poisson bracket on $S(\mathfrak{g})$ is exactly the first-order 1-parameter formal deformation from $S(\mathfrak{g})$ to $U(\mathfrak{g})$.

Remark 4.10. In fact we can find the expressing of the $t^{2}$ term in the star-product. According to [10] Remark 4.7, for any $a, b \in S(\mathfrak{g})$, the $t^{2}$ term is

$$
m_{2}(a, b):=\frac{1}{8} c_{i j}^{s} c_{k l}^{t} X_{s} \cdot X_{t} \cdot \partial^{i} \partial^{k} a \cdot \partial^{j} \partial^{l} b+\frac{1}{12} c_{k s}^{t} c_{j i}^{s} X_{t} \cdot\left(\partial^{k} \partial^{j} a \cdot \partial^{i} b+\partial^{i} a \cdot \partial^{k} \partial^{j} b\right)
$$

Now we can give another proof of Lemma 3.3

Proposition 4.2 (Lemma3.3). We can define a 2-cochain $\phi \in C^{2}(S(\mathfrak{g}), S(\mathfrak{g}))$ as follows: for any $a, b \in S(\mathfrak{g})$

$$
\phi(a, b):=\frac{1}{2} c_{i j}^{s} c_{k l}^{t} X_{s} \cdot X_{t} \cdot \partial^{i} \partial^{k} a \cdot \partial^{j} \partial^{l} b+\frac{1}{3} c_{k s}^{t} c_{j i}^{s} X_{t} \cdot\left(\partial^{k} \partial^{j} a \cdot \partial^{i} b+\partial^{i} a \cdot \partial^{k} \partial^{j} b\right) .
$$

Then for any $a, b, c \in S(\mathfrak{g})$ we have

$$
\{a,\{b, c\}\}-\{\{a, b\}, c\}+\left(d_{H} \phi\right)(a, b, c)=0 .
$$

Proof. The result is clear in the framework of deformation theory (see Appendix C). Let $m=*_{t}$ be the starproduct. Compare Proposition (4.1), Equation (28) and Equation (C.3) we get

$$
P=2 m_{1} \text { and } \phi=4 m_{2} \text {. }
$$

where we denote the Poisson bracket on $S(\mathfrak{g})$ by $P$ too.

Since we know from the definition that the star-product is associative, by Proposition C.2 and in particular Equation (C.10) we get

$$
m_{1} \circ m_{1}+\mathrm{d}_{\mathrm{H}} m_{2}=0
$$

hence

$$
P \circ P+\mathrm{d}_{\mathrm{H}} \phi=0
$$

and this is exactly Equation (30).

If we restrict ourselves to the invariant subalgebra $I(\mathfrak{g})=S(\mathfrak{g})^{\mathfrak{g}}$, then we have the following well-known result about the Poisson bracket:

Proposition $4.3([16])$. The Poisson bracket vanishes on $I(\mathfrak{g})$. In other words, for any $a, b \in I(\mathfrak{g})$, we have

$$
\{a, b\}=0 \text {. }
$$

Proof. This result comes from the definition of $I(\mathfrak{g})$. In fact

$$
\begin{aligned}
\{a, b\} & =c_{i j}^{k} X_{k}\left(\partial^{i} a\right)\left(\partial^{j} b\right) \\
& =\left(c_{i j}^{k} X_{k} \partial^{i} a\right)\left(\partial^{j} b\right) \\
& =\left(\operatorname{ad} X_{j}(a)\right)\left(\partial^{j} b\right) .
\end{aligned}
$$

Since $a \in I(\mathfrak{g})$, we know that $\operatorname{ad} X_{j}(a)=0$ for any $X_{j}$, as a result, $\{a, b\}=0$.

On the other hand, we can easily find the image of $\mathrm{I}_{\mathrm{PBW}}$ restricted on $I(\mathfrak{g})$. 
Proposition 4.4 ([17]). The image of $I(\mathfrak{g})$ under the Poincaré-Birkhoff-Witt map $I_{P B W}$ is exactly $Z_{t}(\mathfrak{g})$, the center of $U_{t}(\mathfrak{g})$. In other words,

$$
I_{P B W}: I(\mathfrak{g}) \rightarrow Z_{t}(\mathfrak{g})
$$

is an isomorphism between vector spaces.

Proof. Keep in mind that $\mathrm{I}_{\mathrm{PBW}}: S(\mathfrak{g}) \rightarrow U_{t}(\mathfrak{g})$ is an isomorphism between $\mathfrak{g}$-vector spaces,i.e. it is compatible with the $\mathfrak{g}$-actions.

Remark 4.11. Proposition 4.3 and Proposition 4.4tell us that the first-order 1-parameter formal deformation from $I(\mathfrak{g})$ to $Z(\mathfrak{g})$ is zero.

In fact we have the much deeper Duflo's isomorphism theorem:

Theorem 4.5 ([6], [18] Section 8, [1] and [3]). There exists an algebraic isomorphism:

$$
\operatorname{Duf}: I(\mathfrak{g}) \rightarrow Z(\mathfrak{g})
$$

We do not give the proof here. Interested readers can check the references above.

Remark 4.12. In general, the map Duf will be different from the Poincaré-Birkhoff-Witt map $\mathrm{I}_{\mathrm{PBW}}$ in Proposition 4.4 although they have the same domain and image.

Remark 4.13. Since $Z(\mathfrak{g})$ is isomorphic to $Z_{t}(\mathfrak{g})$ as algebras, the map Duf can be easily generalized to the map $\operatorname{Duf}_{t}: I(\mathfrak{g}) \rightarrow Z_{t}(\mathfrak{g})$ for any $t$.

B. The 1-parameter formal deformation from $\widetilde{\mathcal{C}_{\tau}}(\mathfrak{g})$ to $\widetilde{\mathcal{Q}_{\tau}}(\mathfrak{g})$ and the noncommutative Poisson bracket

We can generalize the constructions of $S(\mathfrak{g})$ to $\widetilde{\mathcal{C}_{\tau}}(\mathfrak{g})$ in this subsection.

Definition 4.7. We define the algebra $\widetilde{\mathcal{Q}_{\tau}^{t}}(\mathfrak{g})$ as

$$
\widetilde{\mathcal{Q}_{\tau}^{t}}(\mathfrak{g}):=\operatorname{End} V_{\tau} \otimes U_{t}(\mathfrak{g}) .
$$

Moreover, we define

$$
\mathcal{Q}_{\tau}^{t}(\mathfrak{g}):=\left(\operatorname{End} V_{\tau} \otimes U_{t}(\mathfrak{g})\right)^{G} .
$$

By definition, we have $\widetilde{\mathcal{Q}_{\tau}^{0}}(\mathfrak{g})=\widetilde{\mathcal{C}_{\tau}}(\mathfrak{g}), \mathcal{Q}_{\tau}^{0}(\mathfrak{g})=\mathcal{C}_{\tau}(\mathfrak{g})$ and for any $t \neq 0$ we have $\widetilde{\mathcal{Q}_{\tau}^{t}}(\mathfrak{g}) \cong \widetilde{\mathcal{Q}_{\tau}}(\mathfrak{g}), \mathcal{Q}_{\tau}^{t}(\mathfrak{g}) \cong$ $\mathcal{Q}_{\tau}(\mathfrak{g})$.

We also have the Poincaré-Birkhoff-Witt map on the family algebras:

Definition 4.8. The Poincaré-Birkhoff-Witt map $\mathrm{F}_{\mathrm{PBW}}$ on family algebras is defined to be $\mathrm{Id} \otimes \mathrm{I}_{\mathrm{PBW}}$. In other words:

$$
\begin{aligned}
\mathrm{F}_{\mathrm{PBW}}: \widetilde{\mathcal{C}_{\tau}}(\mathfrak{g}) & \longrightarrow \widetilde{\mathcal{Q}_{\tau}^{t}}(\mathfrak{g}) \\
A_{i} \otimes a^{i} & \longmapsto A_{i} \otimes \operatorname{IPBW}\left(a^{i}\right) .
\end{aligned}
$$

$\mathrm{F}_{\mathrm{PBW}}$ is an isomorphism between $\mathfrak{g}$-vector spaces.

As $\mathrm{I}_{\mathrm{PBW}}, \mathrm{F}_{\mathrm{PBW}}$ is not an algebraic isomorphism either. Nevertheless it can also pull back the product on $\widetilde{\mathcal{Q}_{\tau}^{t}}(\mathfrak{g})$ to $\widetilde{\mathcal{C}_{\tau}}(\mathfrak{g})$ :

Definition 4.9. The star-product $*_{t}$ on $\widetilde{\mathcal{C}_{\tau}}(\mathfrak{g})$ is defined to the pull-back of the product on $\widetilde{\mathcal{Q}_{\tau}^{t}}(\mathfrak{g})$ via the map $\mathrm{F}_{\mathrm{PBW}}$. In other words, for any $\mathcal{A}, \mathcal{B} \in \widetilde{\mathcal{C}_{\tau}}(\mathfrak{g})$

$$
\mathcal{A} *_{t} \mathcal{B}:=\mathrm{F}_{\mathrm{PBW}}^{-1}\left(\mathrm{~F}_{\mathrm{PBW}}(\mathcal{A}) \cdot \mathrm{F}_{\mathrm{PBW}}(\mathcal{B})\right) .
$$

Moreover, if we write $\mathcal{A}=A_{i} \otimes a^{i}$ and $\mathcal{B}=B_{j} \otimes b^{j}$, then

$$
\left(A_{i} \otimes a^{i}\right) *_{t}\left(B_{j} \otimes b^{j}\right)=A_{i} B_{j} \otimes\left(a^{i} *_{t} b^{j}\right) .
$$


Therefore the map $\mathrm{F}_{\mathrm{PB}}$ gives an algebraic isomorphism

$$
\mathrm{F}_{\mathrm{PBW}}:\left(\widetilde{\mathcal{C}_{\tau}}(\mathfrak{g}), *_{t}\right) \stackrel{\sim}{\longrightarrow}\left(\widetilde{\mathcal{Q}_{\tau}^{t}}(\mathfrak{g}), \cdot\right) .
$$

Hence we can identify $\widetilde{\mathcal{Q}_{\tau}^{t}}(\mathfrak{g})$ with $\left(\widetilde{\mathcal{C}_{\tau}}(\mathfrak{g}), * t\right)$, especially we can identify $\widetilde{\mathcal{Q}_{\tau}}(\mathfrak{g})$ with $\left(\widetilde{\mathcal{C}_{\tau}}(\mathfrak{g}), *\right)$.

For the star-product on $\widetilde{\mathcal{C}_{\tau}}(\mathfrak{g})$, we also have

Proposition 4.6. We can write the star-product $*_{t}$ on $\widetilde{\mathcal{C}_{\tau}}(\mathfrak{g})$ as

$$
\mathcal{A} *_{t} \mathcal{B}=\mathcal{A B}+\frac{t}{2}\{\mathcal{A}, \mathcal{B}\}+O\left(t^{2}\right)
$$

In other words, the Poisson bracket on $\widetilde{\mathcal{C}_{\tau}}(\mathfrak{g})$ is exactly the first-order 1-parameter formal deformation from $\widetilde{\mathcal{C}_{\tau}}(\mathfrak{g})$ to $\widetilde{\mathcal{Q}_{\tau}}(\mathfrak{g})$.

Proof. This is just a combination of the definition of star-product (Definition 4.9), the definition of noncommutative Poisson bracket (Definition 3.4) and Proposition 4.1

Remark 4.14. By now, the results in this subsection exemplified the slogan "the deformation theory of an algebra $A$ is the same as that of the matrix algebra $\operatorname{Mat}_{n \times n}(A)$." However, when restrict to the invariant subalgebras, these two become different.

If we restrict ourselves to the family algebra $\mathcal{C}_{\tau}(\mathfrak{g})$, i.e. the invariant subalgebra of $\widetilde{\mathcal{C}_{\tau}}(\mathfrak{g})$, we get the follow proposition which is similar to Proposition 4.4

Proposition 4.7. The image of $\mathcal{C}_{\tau}(\mathfrak{g})$ under the Poincaré-Birkhoff-Witt map $F_{P B W}$ is exactly $\mathcal{Q}_{\tau}^{t}(\mathfrak{g})$, the invariant subalgebra of $\widetilde{\mathcal{Q}_{\tau}^{t}}(\mathfrak{g})$. In other words,

$$
F_{P B W}: \mathcal{C}_{\tau}(\mathfrak{g}) \rightarrow \mathcal{Q}_{\tau}^{t}(\mathfrak{g})
$$

is an isomorphism between vector spaces.

Proof. Just remember that $\mathrm{F}_{\mathrm{PBW}}: \widetilde{\mathcal{C}_{\tau}}(\mathfrak{g}) \rightarrow \widetilde{\mathcal{Q}_{\tau}^{t}}(\mathfrak{g})$ is an isomorphism between $\mathfrak{g}$-vector spaces,i.e. it is compatible with the $\mathfrak{g}$-actions.

Now it is natural to ask for the corresponding result of Proposition 4.3 and the Duflo's isomorphism theorem 4.5 on family algebras.

In fact, in Theorem 5.4 of this paper we will prove that the noncommutative Poisson bracket vanishes in the Hochschild cohomology. The generalization of Duflo's isomorphism theorem to family algebras is still an open problem, see Section DI.

\section{THE VANISHING OF THE NONCOMMUTATIVE POISSON BRACKET IN $\operatorname{HH}^{2}\left(\mathcal{C}_{\tau}(\mathfrak{g})\right)$}

\section{A. The twisted gradient map}

In this section we focus on the classical family algebra $\mathcal{C}_{\tau}(\mathfrak{g})$ and the matrix algebra $\widetilde{\mathcal{C}_{\tau}}(\mathfrak{g})$.

Definition 5.10 (The twisted gradient map). We define a map $\nabla: \widetilde{\mathcal{C}_{\tau}}(\mathfrak{g}) \rightarrow \widetilde{\mathcal{C}_{\tau}}(\mathfrak{g})$ as follows: Fix a basis $X_{k}$ of $\mathfrak{g}$. Let $\mathcal{A}=A_{i} \otimes a^{i} \in \widetilde{\mathcal{C}_{\tau}}(\mathfrak{g})$, then

$$
\nabla\left(A_{i} \otimes a^{i}\right):=A_{i} \tau\left(X_{k}\right) \otimes \partial^{k}\left(a^{i}\right) .
$$

Notice that $\widetilde{\mathcal{C}_{\tau}}(\mathfrak{g})$ is nothing but a matrix algebra with entries in $S(\mathfrak{g})$. In the form of matrices,

$$
\nabla(\mathcal{A})=\partial^{k}(\mathcal{A}) \tau\left(X_{k}\right) .
$$

Hence $\nabla$ is a first-order differential operator on $\widetilde{\mathcal{C}_{\tau}}(\mathfrak{g})$ and we call it the twisted gradient map.

From Equation (43) it is not difficult to see that the map $\nabla$ dose not depend on the concrete expression of $\mathcal{A} \in \widetilde{\mathcal{C}_{\tau}}(\mathfrak{g})$ as $A_{i} \otimes a^{i}$.

To show $\nabla$ is a well-defined map, it is now sufficient to prove the following proposition:

Proposition 5.1. The map $\nabla: \widetilde{\mathcal{C}_{\tau}}(\mathfrak{g}) \rightarrow \widetilde{\mathcal{C}_{\tau}}(\mathfrak{g})$ is independent of the choice of the basis of $\mathfrak{g}$. 
Proof. We need to do some computations. Let $\tilde{X}_{j}$ be another basis of $\mathfrak{g}$. Then

$$
\tilde{X}_{j}=T_{j}^{k} X_{k}
$$

where $T_{j}^{k}$ is the transition matrix. Then, let $\tilde{\partial}^{j}$ be the partial derivation with respect to $\tilde{X}_{j}$, we have

$$
\tilde{\partial}^{j}=\left(T^{-1}\right)_{k}^{j} \partial^{k}
$$

Let $\tilde{\nabla}$ be the $\nabla$ map under the basis $\tilde{X}_{j}$, for $A_{i} \otimes a^{i} \in \widetilde{\mathcal{C}_{\tau}}(\mathfrak{g})$, we have

$$
\begin{aligned}
\tilde{\nabla}\left(A_{i} \otimes a^{i}\right) & =A_{i} \tau\left(\tilde{X}_{j}\right) \otimes \tilde{\partial}^{j}\left(a^{i}\right) \\
& =A_{i} \tau\left(T_{j}^{k} X_{k}\right) \otimes\left(T^{-1}\right)_{l}^{j} \partial^{l}\left(a^{i}\right) .
\end{aligned}
$$

The constant $\left(T^{-1}\right)_{l}^{j}$ can be moved to the first component, hence

$$
\text { the above } \begin{aligned}
& =T_{j}^{k}\left(T^{-1}\right)_{l}^{j} A_{i} \tau\left(X_{k}\right) \otimes \partial^{l}\left(a^{i}\right) \\
& =\delta_{l}^{k} A_{i} \tau\left(X_{k}\right) \otimes \partial^{l}\left(a^{i}\right) \\
& =A_{i} \tau\left(X_{k}\right) \otimes \partial^{k}\left(a^{i}\right) \\
& =\nabla\left(A_{i} \otimes a^{i}\right)
\end{aligned}
$$

So $\nabla$ is invariant under the change of basis of $\mathfrak{g}$.

The map $\nabla$ is obviously $\mathbb{C}$-linear, moreover it has the following important property:

Proposition 5.2. The image under $\nabla$ of the subalgebra $\mathcal{C}_{\tau}(\mathfrak{g})$ is contained in $\mathcal{C}_{\tau}(\mathfrak{g})$ itself.

Proof. The proof requires some careful computations.

Let $A_{i} \otimes a^{i} \in \mathcal{C}_{\tau}(\mathfrak{g})$, then

$$
\nabla\left(A_{i} \otimes a^{i}\right)=A_{i} \tau\left(X_{k}\right) \otimes \partial^{k}\left(a^{i}\right) .
$$

By the infinitesimal test of the classical family algebra as in Proposition 2.2, it is sufficient to show that for $X_{j}$ which is one of the basis of $\mathfrak{g}$, we have

$$
\left[\tau\left(X_{j}\right), A_{i} \tau\left(X_{k}\right)\right] \otimes \partial^{k} a^{i}=A_{i} \tau\left(X_{k}\right) \otimes\left\{\partial^{k} a^{i}, X_{j}\right\}
$$

In fact

the left hand side of Equation (44)

$$
\begin{aligned}
& =\left[\tau\left(X_{j}\right), A_{i}\right] \tau\left(X_{k}\right) \otimes \partial^{k} a^{i}+A_{i}\left[\tau\left(X_{j}\right), \tau\left(X_{k}\right)\right] \otimes \partial^{k} a^{i} \\
& =\nabla\left(\left[\tau\left(X_{j}\right), A_{i}\right] \otimes a^{i}\right)+A_{i}\left[\tau\left(X_{j}\right), \tau\left(X_{k}\right)\right] \otimes \partial^{k} a^{i} .
\end{aligned}
$$

To make the following computation more clear, let us denote:

$$
\begin{aligned}
\alpha & :=\nabla\left(\left[\tau\left(X_{j}\right), A_{i}\right] \otimes a^{i}\right), \\
\beta & :=A_{i}\left[\tau\left(X_{j}\right), \tau\left(X_{k}\right)\right] \otimes \partial^{k} a^{i} .
\end{aligned}
$$

First we study $\alpha$. Since $A_{i} \otimes a^{i} \in \mathcal{C}_{\tau}(\mathfrak{g})$, by Proposition 2.2 we have:

$$
\alpha=\nabla\left(\left[\tau\left(X_{j}\right), A_{i}\right] \otimes a^{i}\right)=\nabla\left(A_{i} \otimes\left\{a^{i}, X_{j}\right\}\right)
$$

From the definition of the Poisson bracket on $S(\mathfrak{g})$, we know that

$$
\left\{a^{i}, X_{j}\right\}=c_{s l}^{r} X_{r} \cdot \partial^{s} a^{i} \cdot \partial^{l} X_{j}=c_{s l}^{r} X_{r} \cdot \partial^{s} a^{i} \cdot \delta_{j}^{l}=c_{s j}^{r} X_{r} \partial^{s} a^{i} .
$$

Therefore

$$
\begin{aligned}
\alpha & =\nabla\left(A_{i} \otimes c_{s j}^{r} X_{r} \cdot \partial^{s} a^{i}\right) \\
& =A_{i} \tau\left(X_{l}\right) \otimes \partial^{l}\left(c_{s j}^{r} X_{r} \cdot \partial^{s} a^{i}\right) \\
& =A_{i} \tau\left(X_{l}\right) \otimes c_{s j}^{r}\left(\partial^{l}\left(X_{r}\right) \partial^{s} a^{i}+X_{r} \cdot \partial^{l} \partial^{s} a^{i}\right) \\
& =A_{i} \tau\left(X_{l}\right) \otimes c_{s j}^{r} \delta_{r}^{l} \cdot \partial^{s} a^{i}+A_{i} \tau\left(X_{l}\right) \otimes c_{s j}^{r} X_{r} \cdot \partial^{l} \partial^{s} a^{i} \\
& =A_{i} \tau\left(X_{r}\right) \otimes c_{s j}^{r} \partial^{s} a^{i}+A_{i} \tau\left(X_{l}\right) \otimes c_{s j}^{r} X_{r} \cdot \partial^{s} \partial^{l} a^{i} .
\end{aligned}
$$


Nevertheless, we have

$$
c_{s j}^{r} X_{r} \cdot \partial^{s} \partial^{l} a^{i}=\left\{\partial^{l} a^{i}, X_{j}\right\}
$$

As a result

$$
\alpha=A_{i} \tau\left(X_{r}\right) \otimes c_{s j}^{r} \partial^{s} a^{i}+A_{i} \tau\left(X_{l}\right) \otimes\left\{\partial^{l} a^{i}, X_{j}\right\}
$$

As for $\beta$, we know

$$
\begin{aligned}
\beta & =A_{i}\left[\tau\left(X_{j}\right), \tau\left(X_{k}\right)\right] \otimes \partial^{k} a^{i} \\
& =A_{i} \tau\left(\left[X_{j}, X_{k}\right]\right) \otimes \partial^{k} a^{i} .
\end{aligned}
$$

We know that $\left[X_{j}, X_{k}\right]=c_{j k}^{r} X_{r}$ hence

$$
\tau\left(\left[X_{j}, X_{k}\right]\right)=\tau\left(c_{j k}^{r} X_{r}\right)=c_{j k}^{r} \tau\left(X_{r}\right)=-c_{k j}^{r} \tau\left(X_{r}\right) .
$$

As a result

$$
\begin{aligned}
\beta & =A_{i} \tau\left(\left[X_{j}, X_{k}\right]\right) \otimes \partial^{k} a^{i} \\
& =-A_{i} c_{k j}^{r} \tau\left(X_{r}\right) \otimes \partial^{k} a^{i} \\
& =-A_{i} \tau\left(X_{r}\right) \otimes c_{k j}^{r} \partial^{k} a^{i} .
\end{aligned}
$$

Put Equation (45) and (46) together we get

the left hand side of (44)

$$
\begin{aligned}
& =\alpha+\beta \\
& =A_{i} \tau\left(X_{r}\right) \otimes c_{s j}^{r} \partial^{s} a^{i}+A_{i} \tau\left(X_{l}\right) \otimes\left\{\partial^{l} a^{i}, X_{j}\right\}-A_{i} \tau\left(X_{r}\right) \otimes c_{k j}^{r} \partial^{k} a^{i} \\
& =A_{i} \tau\left(X_{l}\right) \otimes\left\{\partial^{l} a^{i}, X_{j}\right\} \\
& =\text { the right hand side of (44). }
\end{aligned}
$$

This finishes the proof.

Now with Proposition 5.2 we can say that the twisted gradient map $\nabla$ is a $\mathbb{C}$-linear map from $\mathcal{C}_{\tau}(\mathfrak{g})$ to $\mathcal{C}_{\tau}(\mathfrak{g})$. In other words, $\nabla$ can be considered as a Hochschild 1-cochain. see Appendix A for a review of Hochschild cohomology.

Remark 5.15. In general $\nabla$ is not a Hochschild 1-cocycle, see Theorem 5.4 below.

Before moving on to the next section, we give a direct application of the map $\nabla$.

Corollary 5.3 ([14] Section 1). When the Lie algebra $\mathfrak{g}$ is semisimple and $\tau$ is a nontrivial irreducible representation, the classical family algebra $\mathcal{C}_{\tau}(\mathfrak{g})$ is more than $I(\mathfrak{g})$, i.e. $I(\mathfrak{g}) \varsubsetneqq \mathcal{C}_{\tau}(\mathfrak{g})$, and we also have $Z(\mathfrak{g}) \varsubsetneqq \mathcal{Q}_{\tau}(\mathfrak{g})$.

Proof. Let Cas be the quadratic Casimir element in $I(\mathfrak{g})$, deg Cas $=2$. Then by Proposition 5.2, we know that $\nabla(\mathrm{Cas}) \in \mathcal{C}_{\tau}(\mathfrak{g})$ but $\operatorname{deg} \nabla(\mathrm{Cas})=1$. Since $\tau$ is nontrivial we know that $\nabla(\mathrm{Cas}) \neq 0$. On the other hand, since $\mathfrak{g}$ is semisimple, there is no nonzero degree- 1 element in $I(\mathfrak{g})$, therefore $\nabla($ Cas $) \notin I(\mathfrak{g})$ hence $I(\mathfrak{g}) \varsubsetneqq \mathcal{C}_{\tau}(\mathfrak{g})$.

Since there is a PBW map F FBW $: \mathcal{C}_{\tau}(\mathfrak{g}) \rightarrow \mathcal{Q}_{\tau}(\mathfrak{g})$ which maps $I(\mathfrak{g})$ to $Z(\mathfrak{g})$, we know that $Z(\mathfrak{g}) \varsubsetneqq \mathcal{Q}_{\tau}(\mathfrak{g})$.

Remark 5.16. In fact, in Example 2.2 the element $M$ is obtained in the same way as $\nabla$ (Cas) in the above corollary.

Remark 5.17. The definition of $\nabla$ is motivated by the construction of the element $M_{P}$ defined in Section 1 of [14]. Nevertheless in that paper $M_{P}$ is defined only for $P \in I(\mathfrak{g})$ and here we extend the domain to all $\mathcal{C}_{\tau}(\mathfrak{g})$.

\section{B. The relation between the twisted gradient map and the Poisson bracket}

In this subsection, we build up the relation between $\nabla$ and the Poisson bracket $P$.

First we review some notations of Hochshchild cohomology. Notice that $\nabla: \mathcal{C}_{\tau}(\mathfrak{g}) \rightarrow \mathcal{C}_{\tau}(\mathfrak{g})$ is a Hochshchild 1-cochain, i.e.

$$
\nabla \in C^{1}\left(\mathcal{C}_{\tau}(\mathfrak{g}), \mathcal{C}_{\tau}(\mathfrak{g})\right)
$$


Let

$$
\mathrm{d}_{\mathrm{H}}: C^{1}\left(\mathcal{C}_{\tau}(\mathfrak{g}), \mathcal{C}_{\tau}(\mathfrak{g})\right) \rightarrow C^{2}\left(\mathcal{C}_{\tau}(\mathfrak{g}), \mathcal{C}_{\tau}(\mathfrak{g})\right)
$$

be the differential map in the Hochschild complex.

Let $\mathcal{A}, \mathcal{B} \in \mathcal{C}_{\tau}(\mathfrak{g})$. Then by the definition of $\mathrm{d}_{\mathrm{H}}$, we have

$$
\left(\mathrm{d}_{\mathrm{H}} \nabla\right)(\mathcal{A}, \mathcal{B})=\mathcal{A} \nabla(\mathcal{B})-\nabla(\mathcal{A B})+\nabla(\mathcal{A}) \mathcal{B}
$$

The following theorem is the main result of this paper.

Theorem 5.4. For any $\mathcal{A}=A_{i} \otimes a^{i}, \mathcal{B}=B_{j} \otimes b^{j} \in \mathcal{C}_{\tau}(\mathfrak{g})$, we have

$$
\{A, B\}=-\mathcal{A} \nabla(\mathcal{B})+\nabla(\mathcal{A B})-\nabla(\mathcal{A}) \mathcal{B} .
$$

In other words

$$
P+d_{H} \nabla=0
$$

as elements in the Hochschild 2-cochain $C^{2}\left(\mathcal{C}_{\tau}(\mathfrak{g}), \mathcal{C}_{\tau}(\mathfrak{g})\right)$. Therefore the Poisson bracket is a Hochschild coboundary in $C^{2}\left(\mathcal{C}_{\tau}(\mathfrak{g}), \mathcal{C}_{\tau}(\mathfrak{g})\right)$.

Proof. First let us see what is $\nabla(\mathcal{A B})$ :

$$
\begin{aligned}
\nabla(\mathcal{A B}) & =\nabla\left(A_{i} B_{j} \otimes a^{i} b^{j}\right) \\
& =A_{i} B_{j} \tau\left(X_{k}\right) \otimes \partial^{k}\left(a^{i} b^{j}\right) \\
& =A_{i} B_{j} \tau\left(X_{k}\right) \otimes\left(\partial^{k} a^{i}\right) b^{j}+A_{i} B_{j} \tau\left(X_{k}\right) \otimes a^{i}\left(\partial^{k} b^{j}\right)
\end{aligned}
$$

To make the computation more clear, let us denote:

$$
\begin{aligned}
\xi & :=A_{i} B_{j} \tau\left(X_{k}\right) \otimes\left(\partial^{k} a^{i}\right) b^{j}, \\
\eta & :=A_{i} B_{j} \tau\left(X_{k}\right) \otimes a^{i}\left(\partial^{k} b^{j}\right) .
\end{aligned}
$$

Then

$$
\nabla(A B)=\xi+\eta
$$

It is easy to see that $\eta=\mathcal{A} \nabla(\mathcal{B})$. In fact

$$
\eta=A_{i} B_{j} \tau\left(X_{k}\right) \otimes a^{i}\left(\partial^{k} b^{j}\right)=\left(A_{i} \otimes a^{i}\right) \cdot\left(B_{j} \tau\left(X_{k}\right) \otimes \partial^{k} b^{j}\right)=\mathcal{A} \nabla(\mathcal{B}) .
$$

On the other hand, $\xi \neq(\nabla \mathcal{A}) \mathcal{B}$ in general. We know that

$$
\xi=A_{i} B_{j} \tau\left(X_{k}\right) \otimes\left(\partial^{k} a^{i}\right) b^{j}
$$

and

$$
\begin{aligned}
(\nabla \mathcal{A}) \mathcal{B} & =\left(A_{i} \tau\left(X_{k}\right) \otimes \partial^{k} a^{i}\right) \cdot\left(B_{j} \otimes b^{j}\right) \\
& =A_{i} \tau\left(X_{k}\right) B_{j} \otimes\left(\partial^{k} a^{i}\right) b^{j} .
\end{aligned}
$$

Therefore

$$
\begin{aligned}
\xi-(\nabla \mathcal{A}) \mathcal{B} & =\left(A_{i} B_{j} \tau\left(X_{k}\right)-A_{i} \tau\left(X_{k}\right) B_{j}\right) \otimes\left(\partial^{k} a^{i}\right) b^{j} \\
& =A_{i}\left[B_{j}, \tau\left(X_{k}\right)\right] \otimes\left(\partial^{k} a^{i}\right) b^{j} .
\end{aligned}
$$

We need to further simplify the expression $A_{i}\left[B_{j}, \tau\left(X_{k}\right)\right] \otimes\left(\partial^{k} a^{i}\right) b^{j}$. In fact we have the following lemma

Lemma 5.5. any $\mathcal{A}=A_{i} \otimes a^{i}, \mathcal{B}=B_{j} \otimes b^{j} \in \mathcal{C}_{\tau}(\mathfrak{g})$, we have

$$
A_{i}\left[B_{j}, \tau\left(X_{k}\right)\right] \otimes\left(\partial^{k} a^{i}\right) b^{j}=A_{i} B_{j} \otimes\left\{a^{i}, b^{j}\right\}=\{\mathcal{A}, \mathcal{B}\} .
$$


Proof of Lemma 5.5. First by Lemma 2.1 we have

$$
A_{i}\left[B_{j}, \tau\left(X_{k}\right)\right] \otimes\left(\partial^{k} a^{i}\right) b^{j}=\left(A_{i} \otimes \partial^{k} a^{i}\right) \cdot\left(\left[B_{j}, \tau\left(X_{k}\right)\right] \otimes b^{j}\right) .
$$

Since $\mathcal{B}=B_{j} \otimes b^{j}$ is contained in $\mathcal{C}_{\tau}(\mathfrak{g})$, by Proposition 2.2 we know that

$$
\begin{aligned}
& \left(A_{i} \otimes \partial^{k} a^{i}\right) \cdot\left(B_{j} \otimes\left\{X_{k}, b^{j}\right\}\right) \\
= & A_{i} B_{j} \otimes\left(\partial^{k} a^{i}\right) \cdot\left\{X_{k}, b^{j}\right\} \\
= & A_{i} B_{j} \otimes\left\{a^{i}, b^{j}\right\} \\
= & \{\mathcal{A}, \mathcal{B}\} .
\end{aligned}
$$

This proves Lemma 5.5 .

By Lemma 5.5 and Equation (53) we have

$$
\xi=\{\mathcal{A}, \mathcal{B}\}+(\nabla \mathcal{A}) \mathcal{B}
$$

Put equations (51), 52) and (55) together, we have:

$$
\begin{aligned}
& \nabla(\mathcal{A B})-\mathcal{A} \nabla(\mathcal{B})-\nabla(\mathcal{A}) \mathcal{B} \\
= & \xi+\eta-\mathcal{A} \nabla(\mathcal{B})-\nabla(\mathcal{A}) \mathcal{B} \\
= & \{\mathcal{A}, \mathcal{B}\}+(\nabla \mathcal{A}) \mathcal{B}+\mathcal{A} \nabla(\mathcal{B})-\mathcal{A} \nabla(\mathcal{B})-(\nabla \mathcal{A}) \mathcal{B} \\
= & \{\mathcal{A}, \mathcal{B}\} .
\end{aligned}
$$

This finishes the proof of Theorem 5.4

Caution 5.3. Although both the twisted gradient map $\nabla$ and the Poisson bracket $P$ can be defined on the larger algebra $\widetilde{\mathcal{C}_{\tau}}(\mathfrak{g})$, we do not have the relation

$$
\{\mathcal{A}, \mathcal{B}\}=-\mathrm{d}_{\mathrm{H}} \nabla(\mathcal{A}, \mathcal{B})
$$

for any $\mathcal{A}, \mathcal{B} \in \widetilde{\mathcal{C}_{\tau}}(\mathfrak{g})$. Actually in the proof of Lemma 5.5 we see that it is necessary to have $\mathcal{B} \in \mathcal{C}_{\tau}(\mathfrak{g})$.

From the view point of deformation theory (Proposition C.3), we have the following corollary.

Corollary 5.6. The 1-parameter formal deformation from $\mathcal{C}_{\tau}(\mathfrak{g})$ to $\mathcal{Q}_{\tau}(\mathfrak{g})$ is infinitesimally trivial.

Proof. We know in Proposition 4.6 that the first order 1-parameter formal deformation $m_{1}$ is $\frac{1}{2} P$, therefore this corollary is just a direct consequence of Theorem 5.4 .

Using Theorem 5.4 we can also give an alternative proof of Proposition 3.4 as follows.

Corollary 5.7 (Proposition 3.4). For any $\mathcal{A}, \mathcal{B} \in \mathcal{C}_{\tau}(\mathfrak{g})$, we have that $\{\mathcal{A}, \mathcal{B}\}$ is still in $\mathcal{C}_{\tau}(\mathfrak{g})$.

Proof. In the proof of Theorem 5.4, we do not require a priori that $\{\mathcal{A}, \mathcal{B}\} \in \mathcal{C}_{\tau}(\mathfrak{g})$. Now by Proposition 5.2 we know that $\nabla$ maps $\mathcal{C}_{\tau}(\mathfrak{g})$ to $\mathcal{C}_{\tau}(\mathfrak{g})$ and from Theorem 5.4 we also know that

$$
\{\mathcal{A}, \mathcal{B}\}=-\mathrm{d}_{\mathrm{H}} \nabla(\mathcal{A}, \mathcal{B})
$$

hence we get the result we want.

\section{Digression: An alternative of the twisted gradient map}

In this subsection we want to show that the twisted gradient map $\nabla$ defined in Definition 5.10 is NOT the unique map which satisfies

$$
\{\mathcal{A}, \mathcal{B}\}=-\mathrm{d}_{\mathrm{H}} \nabla(\mathcal{A}, \mathcal{B}) .
$$

In fact we define a map $\nabla^{\prime}: \widetilde{\mathcal{C}_{\tau}}(\mathfrak{g}) \rightarrow \widetilde{\mathcal{C}_{\tau}}(\mathfrak{g})$ to be

$$
\nabla^{\prime}\left(A_{i} \otimes a^{i}\right):=\tau\left(X_{k}\right) A_{i} \otimes \partial^{k} a^{i} .
$$

Similar to Proposition 5.2, we can check that $\nabla^{\prime}$ also maps $\mathcal{C}_{\tau}(\mathfrak{g})$ to $\mathcal{C}_{\tau}(\mathfrak{g})$. 
Remark 5.18. The difference between the definition of $\nabla$ and $\nabla^{\prime}$ is: for $\nabla$, the matrix $\tau\left(X_{k}\right)$ is multiplied from the right; while for $\nabla^{\prime}$, the matrix $\tau\left(X_{k}\right)$ is multiplied from the left.

In general $\nabla^{\prime} \neq \nabla$ and we want to find their difference. First we define the first Chern class on $\widetilde{\mathcal{C}_{\tau}}(\mathfrak{g})$ following [3] Section 1.1.

Definition 5.11. The first Chern class $c_{1}$ is a map $\widetilde{\mathcal{C}_{\tau}}(\mathfrak{g}) \rightarrow \widetilde{\mathcal{C}_{\tau}}(\mathfrak{g}), c_{1}:=\operatorname{tr}(\operatorname{ad})$. More precisely

$$
\begin{aligned}
c_{1}: \widetilde{\mathcal{C}_{\tau}}(\mathfrak{g}) & \longrightarrow \widetilde{\mathcal{C}_{\tau}}(\mathfrak{g}) \\
A \otimes a & \mapsto A \otimes c_{i j}^{j} \partial^{i} a .
\end{aligned}
$$

It is easy to check that $c_{1}$ is $\mathfrak{g}$-invariant hence $c_{1}$ maps $\mathcal{C}_{\tau}(\mathfrak{g})$ to $\mathcal{C}_{\tau}(\mathfrak{g})$. Moreover, it is also easy to check that the first Chern class is closed in the Hochschild cochain. In other words, $c_{1} \in Z^{1}\left(\widetilde{\mathcal{C}_{\tau}}(\mathfrak{g}), \widetilde{\mathcal{C}_{\tau}}(\mathfrak{g})\right)$ and $\left.c_{1}\right|_{\mathcal{C}_{\tau}(\mathfrak{g})} \in$ $Z^{1}\left(\mathcal{C}_{\tau}(\mathfrak{g}), \mathcal{C}_{\tau}(\mathfrak{g})\right)$. For simplicity we also write $c_{1}$ for the restriction $\left.c_{1}\right|_{\mathcal{C}_{\tau}(\mathfrak{g})}$.

Having the first Chern class, we can express the difference between $\nabla$ and $\nabla^{\prime}$ in $\mathcal{C}_{\tau}(\mathfrak{g})$ :

Proposition 5.8. In the classical family algebra $\mathcal{C}_{\tau}(\mathfrak{g})$ we have

$$
\nabla-\nabla^{\prime}=-c_{1} .
$$

Proof. For any $A_{i} \otimes a^{i} \in \mathcal{C}_{\tau}(\mathfrak{g})$

$$
\begin{aligned}
\nabla\left(A_{i} \otimes a^{i}\right)-\nabla^{\prime}\left(A_{i} \otimes a^{i}\right) & =A_{i} \tau\left(X_{k}\right) \otimes \partial^{k} a^{i}-\tau\left(X_{k}\right) A_{i} \otimes \partial^{k} a^{i} \\
& =\left[A_{i}, \tau\left(X_{k}\right)\right] \otimes \partial^{k} a^{i} \\
& =\partial^{k}\left(\left[A_{i}, \tau\left(X_{k}\right)\right] \otimes a^{i}\right) \text { (We can move the partial derivative out). }
\end{aligned}
$$

Since $A_{i} \otimes a^{i} \in \mathcal{C}_{\tau}(\mathfrak{g})$, we have

$$
\left[A_{i}, \tau\left(X_{k}\right)\right] \otimes a^{i}=A_{i} \otimes\left\{X_{k}, a^{i}\right\}
$$

Therefore

$$
\begin{aligned}
\nabla\left(A_{i} \otimes a^{i}\right)-\nabla^{\prime}\left(A_{i} \otimes a^{i}\right) & =\partial^{k}\left(A_{i} \otimes\left\{X_{k}, a^{i}\right\}\right) \\
& =A_{i} \otimes \partial^{k}\left(\left\{X_{k}, a^{i}\right\}\right) \\
& =A_{i} \otimes \partial^{k}\left(c_{k j}^{l} X_{l} \cdot \partial^{j} a^{i}\right) \\
& =A_{i} \otimes\left(c_{k j}^{k} \partial^{j} a^{i}+c_{k j}^{l} X_{l} \cdot \partial^{k} \partial^{j} a^{i}\right) \\
& =A_{i} \otimes c_{k j}^{k} \partial^{j} a^{i}+A_{i} \otimes c_{k j}^{l} X_{l} \cdot \partial^{k} \partial^{j} a^{i} \\
& =-c_{1}\left(A_{i} \otimes a^{i}\right)+A_{i} \otimes c_{k j}^{l} X_{l} \cdot \partial^{k} \partial^{j} a^{i}
\end{aligned}
$$

Since $c_{k j}^{l}$ is anti symmetric with respect to $k, j$, it is easy to see that

$$
A_{i} \otimes c_{k j}^{l} X_{l} \cdot \partial^{k} \partial^{j} a^{i}=0
$$

Hence we get

$$
\nabla\left(A_{i} \otimes a^{i}\right)-\nabla^{\prime}\left(A_{i} \otimes a^{i}\right)=-c_{1}\left(A_{i} \otimes a^{i}\right)
$$

The next corollary tells us that we can replace $\nabla$ by $\nabla^{\prime}$ in Theorem 5.4

Corollary 5.9. In $\mathcal{C}_{\tau}(\mathfrak{g})$ we have

$$
d_{H} \nabla^{\prime}=d_{H} \nabla=P, \text { the Poisson bracket on } \mathcal{C}_{\tau}(\mathfrak{g}) .
$$

Therefore we can replace $\nabla$ by $\nabla^{\prime}$ in Theorem 5.4

Proof. We know that $\mathrm{d}_{\mathrm{H}} \nabla=P$ and $\nabla-\nabla^{\prime}=-c_{1}$. In Definition 5.11 we also know that $c_{1}$ is closed, i.e. $\mathrm{d}_{\mathrm{H}} c_{1}=0$. Hence we get this corollary.

At the end of this subsection we should point out that although $\nabla^{\prime} \neq \nabla$ in general, they are equal in some important cases. Actually we have the following result.

Proposition 5.10. When $\mathfrak{g}$ is a semisimple Lie algebra, we have $\nabla^{\prime}=\nabla$ in $\mathcal{C}_{\tau}(\mathfrak{g})$.

Proof. We know that for semisimle Lie algebra, the adjoint representation is traceless, in other words

$$
c_{i j}^{i}=0 \text { for any } j .
$$

Therefore $c_{1}=0$ for semisimple $\mathfrak{g}$, hence we get the result. 


\section{FURTHER TOPICS: MACKEY'S ANALOGUE AND THE QUANTIZATION OF THE FAMILY ALGEBRAS}

This section is a survey of further topics. In this section we restrict to the case that $\mathfrak{g}$ is a complex semisimple Lie algebra and the representation $\tau$ to be a simple representation of $\mathfrak{g}$.

In 1975 G. Mackey ([20]) studied the analogies between the representations of a semisimple Lie group $G$ and those of its Cartan motion group $G_{c}$. Later N. Higson find the relation between family algebras and Mackey's analogue in [12] as we have mentioned in the introduction.

Remark 6.19. In fact, Higson introduced the spherical Hecke algebras $\mathcal{R}(\mathfrak{g}, \tau)$ and $\mathcal{R}\left(\mathfrak{g}_{c}, \tau\right)$ respectively. These algebras have the importance that the irreducible $\mathcal{R}(\mathfrak{g}, \tau)$ modules are 1-1 correspondent to irreducible $(\mathfrak{g}, K)$ modules of $G$ with nonzero $\tau$-isotypical component, and the similar result holds for $\mathcal{R}\left(\mathfrak{g}_{c}, \tau\right)$. When $G$ is complex semisimple, Higson proved that the spherical Hecke algebras are isomorphic to the family algebras. For details see [12].

Let $\mathfrak{h} \subset \mathfrak{g}$ be the Cartan subalgebra. Higson also constructed the generalized Harish-Chandra homomorphisms:

$$
\begin{aligned}
& \mathrm{GHC}_{\tau}: \mathcal{R}(\mathfrak{g}, \tau) \rightarrow U(\mathfrak{h}) \\
& \mathrm{GHC}_{\tau, c}: \mathcal{R}\left(\mathfrak{g}_{c}, \tau\right) \rightarrow S(\mathfrak{h})
\end{aligned}
$$

and relates them to the admissible duals of $G$ and $G_{c}$ with minimal $K$-type $\tau$.

The Mackey's analogue for admissible dual of complex semisimple $G$ has the following form:

Theorem 6.1 ([12], Section 8). Under the identification $U(\mathfrak{h}) \cong S(\mathfrak{h})$, the two homomorphisms $G_{H} C_{\tau}$ and $G H C_{\tau, c}$ has the same image.

In the end of [12], Higson proposed the problem of constructing a quantization map $Q$ between $\mathcal{C}_{\tau}(\mathfrak{g})$ and $\mathcal{Q}_{\tau}(\mathfrak{g})$ such that the following diagram commutes.

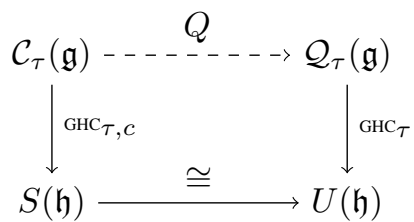

Here $Q$ is a vector space isomorphism but need not to be an algebraic isomorphism.

Remark 6.20. According to Theorem 5.4 and Corollary 5.6, the 1-parameter formal deformation from $\mathcal{C}_{\tau}(\mathfrak{g})$ to $\mathcal{Q}_{\tau}(\mathfrak{g})$ is infinitesimally trivial, which suggests that there exists strong relations between them. Actually Theorem 5.4 can be considered as the first step in the solution of the quantization problem of the family algebras.

\section{ACKNOWLEDGEMENT}

Z.W. wishes to thank Alexandre Kirillov, Vasily Dolgushev, Eckhard Meinrenken, Valery Lunts and in particular Nigel Higson for helpful discussions and comments.

This paper is part of the doctoral dissertation of Z.W. at University of Pennsylvania and Z.W would like to thank his doctoral advisor Jonathan Block for his help on this topic and encouragements.

\section{Appendix A: Hochschild cohomology}

Let us review the theory of Hochschild cohomology in this appendix. For reference see [23] or [3] Section 2.

Let $A$ be an associative $\mathbb{C}$-algebra. The Hochschild complex $C^{\bullet}(A, A)$ is defined as follows:

$$
C^{n}(A, A):=\operatorname{Hom}_{\mathbb{C}}\left(A^{\otimes n}, A\right), n \geqslant 0 .
$$

The differential $\mathrm{d}_{\mathrm{H}}$ is defined on homogeneous elements $f \in C^{n}(A, A)$ by the formula

$$
\begin{aligned}
\left(\mathrm{d}_{\mathrm{H}}(f)\right)\left(a_{0}, a_{1}, \ldots, a_{n}\right): & =a_{0} f\left(a_{1}, \ldots, a_{n}\right)+\sum_{k=1}^{n}(-1)^{k} f\left(a_{0}, \ldots, a_{k-1} a_{k}, \ldots, a_{n}\right) \\
& +(-1)^{n+1} f\left(a_{0}, \ldots, a_{n-1}\right) a_{n} .
\end{aligned}
$$


We see that $\mathrm{d}_{\mathrm{H}} f \in C^{n+1}(A, A)$. Actually we can prove that $\mathrm{d}_{\mathrm{H}} \circ \mathrm{d}_{\mathrm{H}}=0$ therefore $C^{\bullet}(A, A)$ is a cochain complex.

The Hochschild cohomology of $A$ is defined as the cohomology group of the cochain complex $C^{\bullet}(A, A)$, and we denote it by $\mathrm{HH}^{\bullet}(A, A)$ or for short $\mathrm{HH}^{\bullet}(A)$ :

$$
\mathrm{HH}^{n}(A):=\mathrm{H}^{n}\left(C^{\bullet}(A, A)\right) .
$$

Now let us look at the case $n=2$. The following result is easy to get:

Proposition A.1. Let $f \in C^{2}(A, A)=\operatorname{Hom}_{\mathbb{C}}(A \otimes A, A)$. Then $f$ is a 2-coboundary if and only if there exists a $g \in C^{1}(A, A)=\operatorname{Hom}_{\mathbb{C}}(A, A)$ such that for any $a, b \in A$

$$
f(a, b)=a g(b)-g(a b)+g(a) b .
$$

Moreover, $f$ is a 2-cocycle if and only if for any $a, b, \in A$

$$
a f(b, c)-f(a b, c)+f(a, b c)-f(a, b) c=0 .
$$

Proof. Direct check by definition.

\section{Appendix B: The Gerstenhaber bracket on Hochschild cochains and cohomologies}

In this section we give a quick review of the Gerstenhaber bracket. For more details and proofs see [8] or [2] Section 1. For further topics see the survey [4].

First, we define an operation $\circ: C^{k}(A, A) \otimes C^{l}(A, A) \rightarrow C^{k+l-1}(A, A)$. Let $f_{1} \in C^{k}(A, A)$ and $f_{2} \in$ $C^{l}(A, A)$,

$$
\begin{aligned}
& \left(f_{1} \circ f_{2}\right)\left(a_{1}, \ldots, a_{k+l-1}\right):= \\
& \quad=\sum_{i=0}^{k-1}(-1)^{(k-i-1)(l-1)} f_{1}\left(a_{1} \ldots, a_{i}, f_{2}\left(a_{i+1}, \ldots, a_{i+l}\right), a_{i+l+1}, \ldots, a_{k+l-1}\right) .
\end{aligned}
$$

In particular, for 2-cochains we have

Proposition B.1. Let $f_{1}, f_{2} \in C^{2}(A, A)$, then $f_{1} \circ f_{2} \in C^{3}(A, A)$ and is given by

$$
\left(f_{1} \circ f_{2}\right)\left(a_{1}, a_{2}, a_{3}\right)=f_{1}\left(f_{2}\left(a_{1}, a_{2}\right), a_{3}\right)-f_{1}\left(a_{1}, f_{2}\left(a_{2}, a_{3}\right)\right) .
$$

In particular, for $f \in C^{2}(A, A)$ we have

$$
(f \circ f)\left(a_{1}, a_{2}, a_{3}\right)=f\left(f\left(a_{1}, a_{2}\right), a_{3}\right)-f\left(a_{1}, f\left(a_{2}, a_{3}\right)\right) .
$$

Proof. This is exactly the definition.

The Gerstenhaber bracket is defined to be

$$
\left[f_{1}, f_{2}\right]_{\mathrm{G}}:=f_{1} \circ f_{2}-(-1)^{(k-1)(l-1)} f_{2} \circ f_{1} .
$$

The Gerstenhaber bracket is a Lie bracket. In fact we have the following

Theorem B.2. The operation " $\circ "$ gives a pre-Lie algebra structure on $C^{\bullet-1}(A, A)$. Therefore we obtain that $\left(C^{\bullet-1}(A, A),[,]_{G}\right)$ is a graded Lie algebra.

Proof. See [8].

Proposition B.3. Let $f \in C^{2}(A, A)$, then

$$
[f, f]_{G}=2 f \circ f .
$$

Proof. We get this directly from the definitions.

In fact $\mathrm{d}_{\mathrm{H}}$ is an inner derivation under the Gerstenhaber bracket. More precisely, let $\mu: A \otimes A \rightarrow A$ be the multiplication map in $A$. Then $\mu \in C^{2}(A, A)$ and we have the following 
Proposition B.4. For any $f \in C^{k}(A, A)$, we have

$$
d_{H} f=[\mu, f]_{G} \in C^{k+1}(A, A) .
$$

We also have $[\mu, \mu]_{G}=0$.

Proof. Compare the definition of $\mathrm{d}_{\mathrm{H}}$ in Equation (A.2) and the definition of the Gerstenhaber bracket in Equation (B.1) and Equation (B.4). The fact that $[\mu, \mu]_{\mathrm{G}}=0$ is exactly the associativity of $\mu$.

As a result, we have the following theorem:

Theorem B.5. The Gerstenhaber bracket is compatible with the Hochschild differential $d_{H}$. In other words, for any $f_{1} \in C^{k}(A, A)$ and $f_{2} \in C^{l}(A, A)$, we have

$$
d_{H}\left(\left[f_{1}, f_{2}\right]_{G}\right)=\left[d_{H} f_{1}, f_{2}\right]_{G}+(-1)^{k-1}\left[f_{1}, d_{H} f_{2}\right]_{G} .
$$

Therefore the Gerstenhaber bracket reduces to the Hochschild cohomology $\mathrm{HH}^{\bullet-1}(A)$.

Proof. Since $\mathrm{d}_{\mathrm{H}}$ is an inner derivation according to Propostion B.4 Equation B.7) is a consequence of the gradedJacobi identity of the graded Lie algebra $\left(C^{\bullet-1}(A, A),[,]_{\mathrm{G}}\right)$.

\section{Appendix $\mathbf{C}: \mathbf{H H}^{\bullet}(A)$ and the deformations of $A$}

The Hochschild cohomology plays an important role in the deformation theory. Let us summarize some results in the deformation theory of algebras in this appendix. For more details see [9].

Let $A$ be an associative $\mathbb{C}$ algebra (in fact we can replace $\mathbb{C}$ by any field). A deformation of the algebra structure of $A$ means that we fix $A$ as a $\mathbb{C}$-vector space and change the multiplication operation on $A$. Actually there are many kinds of deformations, like analytic, algebraic, formal, global, etc., and in this paper we focus on formal deformation, in particular 1-parameter formal deformation of algebras. In more details let $\mathbb{C}[[t]]$ be the formal power series of $t$ and we define

$$
A[[t]]:=A \otimes_{\mathbb{C}} \mathbb{C}[[t]]
$$

$A[[t]]$ is obviously a $\mathbb{C}[[t]]$-module.

A 1-parameter formal deformation of the algebra structure on $A$ is given by a map

$$
m: A[[t]] \otimes A[[t]] \longrightarrow A[[t]]
$$

where $m$ is required to be $\mathbb{C}[[t]]$-bilinear. So we only need to know the value of $m$ on $A \otimes A$. Moreover we require that $m(a, b) \equiv a b \bmod t$ for $a, b \in A$.

For any $a, b \in A$, we can write $m(a, b)$ as

$$
m(a, b)=a b+\sum_{k=0}^{\infty} t^{k} m_{k}(a, b) .
$$

We see that each $m_{k}$ belongs to $C^{2}(A, A)$.

Remark C.21. The element $t$ is called the deformation parameter. To get an informal idea of deformation theory, we can evaluate at $t=0$ and get the original multiplication on $A$. On the other hand if we evaluate at $t \neq 0$, omit the convergence problem, we get a new binary operation $A \otimes A \rightarrow A$.

Remark C.22. We can also talk about more general formal deformation of $A$, where the algebra $\mathbb{C}[[t]]$ is replaced by a complete local augmented $\mathbb{C}$-algebra, see [5] Section 3.

Moreover we also have formal deformation theory of Lie algebras,see [7] for a detailed introduction.

As a multiplication, the map $m$ needs to satisfy the associativity law.

Theorem C.1 (1-parameter formal deformation, see [9] Chapter I.1). Let $m(a, b)=a b+\sum_{k=0}^{\infty} t^{k} m_{k}(a, b)$ as in Equation (C.3). Then $m$ satisfies the associativity law if and only if for each $k \geqslant 1$, we have

$$
d_{H} m_{k}+\frac{1}{2} \sum_{i=1}^{k-1}\left[m_{i}, m_{k-i}\right]=0 .
$$

If this holds, we say that $m$ gives a formal deformation of $A$. 
Proof. The associativity law tells us that for any $a, b, c \in A$, we have

$$
m(a, m(b, c))-m(m(a, b), c)=0 .
$$

Now consider $m$ as an element in $C^{2}(A[[t]], A[[t]])$, then Equation $(\mathbf{C} .5$ is exactly

$$
[m, m]_{\mathrm{G}}=0 .
$$

We write $m=\mu+\sum_{k=1}^{\infty} t^{k} m_{k}$ where $\mu$ is the original multiplication on $A$. Then because we know

$$
[\mu, f]_{\mathrm{G}}=\mathrm{d}_{\mathrm{H}} f \text { and }[\mu, \mu]_{\mathrm{G}}=0
$$

in Proposition B.4, Equation (C.6) becomes the Maurer-Cartan Equation

$$
\mathrm{d}_{\mathrm{H}}\left(\sum_{k=1}^{\infty} t^{k} m_{k}\right)+\frac{1}{2}\left[\sum_{k=1}^{\infty} t^{k} m_{k}, \sum_{k=1}^{\infty} t^{k} m_{k}\right]_{\mathrm{G}}=0 .
$$

In the expansion of Equation (C.7), we take the $t^{k}$ term and get Equation (C.4).

Corollary C.2 (Infinitesimal deformation). The map $m$ satisfies the associativity law $\bmod t^{2}$ if and only if $d_{H} m_{1}=0$, i.e. for any $a, b, c \in A$, we have

$$
a m_{1}(b, c)-m_{1} a b, c+m_{1}(a, b c)-m_{1}(a, b) c=0 .
$$

If this holds, we say that $m$ gives an infinitesimal deformation of $A$.

Moreover, $m$ satisfies the associativity law $\bmod t^{3}$ if and only if $d_{H} m_{1}=0$ together with

$$
d_{H} m_{2}+\frac{1}{2}\left[m_{1}, m_{1}\right]_{G}=0
$$

The above equation is equivalent to

$$
d_{H} m_{2}+m_{1} \circ m_{1}=0
$$

Proof. This is an direct corollary of Theorem C.1

On the other hand, we need to know when the 1-parameter formal deformation $m$ is trivial. In other words, whether or not we can find an algebraic isomorphism

$$
\theta:(A[[t]], \mu) \longrightarrow(A[[t]], m)
$$

where $\theta$ is $\mathbb{C}[[t]]$-linear and is given by

$$
\theta(a)=a+\sum_{k=1}^{\infty} t^{k} \theta_{k}(a)
$$

The requirement for $\theta$ is for any $a, b \in A$

$$
\theta(a b)=m(\theta(a), \theta(b)) .
$$

The existence of $\theta$ is a complicated issue. Nevertheless as a first step we have:

Proposition C.3 (Infinitesimally trivial deformation). There exists a $\theta_{1} \in C^{1}(A, A)$ such that $\theta=i d+t \theta_{1}$ satisfyies Equation (C.13) $\bmod t^{2}$ if and only if $m_{1} \in B^{2}(A, A)$. If this holds, we say that $m$ is an infinitesimally trivial deformation of $A$.

Proof. We expand both sides of Equation (C.13) and look at the $t$ term we get

$$
\theta_{1}(a b)=\theta_{1}(a) b+a \theta_{1}(b)+m_{1}(a, b)
$$

In other words

$$
m_{1}+\mathrm{d}_{\mathrm{H}} \theta_{1}=0 \text {. }
$$


Further discussion of the triviality of formal deformations involves the concept of gauge equivalence of MaurerCartan elements, see [18] Section 1 or [19] Chapter 13.

[1] A. Alekseev and E. Meinrenken. The non-commutative Weil algebra. Invent. Math., 139(1):135-172, 2000.

[2] Jonathan Block and Ezra Getzler. Quantization of foliations. In Proceedings of the XXth International Conference on Differential Geometric Methods in Theoretical Physics, Vol. 1, 2 (New York, 1991), pages 471-487. World Sci. Publ., River Edge, NJ, 1992.

[3] Damien Calaque and Carlo A. Rossi. Lectures on Duflo isomorphisms in Lie algebra and complex geometry. EMS Series of Lectures in Mathematics. European Mathematical Society (EMS), Zürich, 2011.

[4] Vasiliy Dolgushev, Dmitry Tamarkin, and Boris Tsygan. Formality theorems for Hochschild complexes and their applications. Lett. Math. Phys., 90(1-3):103-136, 2009.

[5] M. Doubek, M. Markl, and P. Zima. Deformation theory (lecture notes). Arch. Math. (Brno), 43(5):333-371, 2007.

[6] Michel Duflo. Opérateurs différentiels bi-invariants sur un groupe de Lie. Ann. Sci. École Norm. Sup. (4), 10(2):265-288, 1977.

[7] A. Fialowski. An example of formal deformations of Lie algebras. In Deformation theory of algebras and structures and applications (Il Ciocco, 1986), volume 247 of NATO Adv. Sci. Inst. Ser. C Math. Phys. Sci., pages 375-401. Kluwer Acad. Publ., Dordrecht, 1988.

[8] Murray Gerstenhaber. The cohomology structure of an associative ring. Ann. of Math. (2), 78:267-288, 1963.

[9] Murray Gerstenhaber. On the deformation of rings and algebras. Ann. of Math. (2), 79:59-103, 1964.

[10] J. Grabowski. Abstract Jacobi and Poisson structures. Quantization and star-products. J. Geom. Phys., 9(1):45-73, 1992.

[11] S. Gutt. An explicit *-product on the cotangent bundle of a Lie group. Lett. Math. Phys., 7(3):249-258, 1983.

[12] Nigel Higson. On the analogy between complex semisimple groups and their Cartan motion groups. In Noncommutative geometry and global analysis, volume 546 of Contemp. Math., pages 137-170. Amer. Math. Soc., Providence, RI, 2011.

[13] Anthony Joseph. Modules for relative Yangians (family algebras) and Kazhdan-Lusztig polynomials. Transform. Groups, 19(1):105-129, 2014.

[14] A. A. Kirillov. Family algebras. Electron. Res. Announc. Amer. Math. Soc., 6:7-20 (electronic), 2000.

[15] A. A. Kirillov. Introduction to family algebras. Mosc. Math. J., 1(1):49-63, 2001.

[16] A. A. Kirillov. Lectures on the orbit method, volume 64 of Graduate Studies in Mathematics. American Mathematical Society, Providence, RI, 2004.

[17] Anthony W. Knapp. Lie groups beyond an introduction, volume 140 of Progress in Mathematics. Birkhäuser Boston Inc., Boston, MA, second edition, 2002.

[18] Maxim Kontsevich. Deformation quantization of Poisson manifolds. Lett. Math. Phys., 66(3):157-216, 2003.

[19] Camille Laurent-Gengoux, Anne Pichereau, and Pol Vanhaecke. Poisson structures, volume 347 of Grundlehren der Mathematischen Wissenschaften [Fundamental Principles of Mathematical Sciences]. Springer, Heidelberg, 2013.

[20] George W. Mackey. On the analogy between semisimple Lie groups and certain related semi-direct product groups. In Lie groups and their representations (Proc. Summer School, Bolyai János Math. Soc., Budapest, 1971), pages 339-363. Halsted, New York, 1975.

[21] Natalia Rozhkovskaya. Quantum family algebras. ProQuest LLC, Ann Arbor, MI, 2002. Thesis (Ph.D.)-University of Pennsylvania.

[22] Matthew Tai. The classical family algebra of the adjoint representation of $s l(n)$. arXiv preprint arXiv:1306.0867, 2013.

[23] Charles A. Weibel. An introduction to homological algebra, volume 38 of Cambridge Studies in Advanced Mathematics. Cambridge University Press, Cambridge, 1994.

[24] Ping Xu. Noncommutative Poisson algebras. Amer. J. Math., 116(1):101-125, 1994. 